\title{
Host Autophagy Response: Friend or Foe in Reproductive Tract Infections
}

\author{
Ankit Shroff, Kanchana Ayyar, Debarchana Saha and Reddy KVR* \\ Division of Molecular Immunology and Microbiology (MIM), National Institute for Research in Reproductive Health, Indian Council of Medical Research, \\ Mumbai - 400 012, India
}

Received: February 28, 2014; Accepted: May 24, 2014; Published: May 26, 2014

*Corresponding author: Reddy KVR, Division of Molecular Immunology and Microbiology (MIM), National Institute for Research in Reproductive Health, Indian Council of Medical Research, Mumbai- 400 012, India, Tel: +91 22 2419 2016; E-mail : reddyk@nirrh.res.in

\begin{abstract}
Autophagy was discovered as a self-degradative process that is induced for balancing the nutrient levels in the cells during the times of starvation. It was found to play an additional housekeeping role in removing misfolded or aggregated proteins, clearing damaged organelles. Recent studies have established autophagy as an important mechanism of the innate immune defense of the host. It acts as a second line of defense for the clearance of the pathogen and is induced by various adaptor proteins like Pattern Recognition Receptors (PRRs). This role of autophagy has been chronicled in many microbial infections such as Group A Streptococcus (GAS), Mycobacterium tuberculosis, Salmonella typhimurium and Enterococcus feacalis. Infections of the reproductive tract form a significant part of the health problems faced by the world and are primarily transmitted through sexual mode. The role of autophagy during reproductive tract infections (RTIs) such as Chlamydiasis, Candidiasis, Trichomoniasis, and Viral Infections like Human Immunodeficiency Virus (HIV), Herpes Simplex Virus (HSV) and Human Papilloma Virus (HPV) have been studied using various in-vitro and in-vivo models. In this review, these findings on the relationships between autophagy and microorganisms causing RTI have been summarized to aid the understanding of the role of autophagy during these infections.
\end{abstract}

Keywords: Autophagy; RTIs; Innate immunity; Host-pathogen relationship

\section{Autophagy}

The term 'autophagy' is derived from Greek, which means 'eating of self'. It is now used as a general term for the degradation of cytoplasmic and pathogenic components through the lysosome mediated pathway. The self-degradative nature of autophagy is essential in times of starvation as an amino acid source. Autophagy is constitutively involved in removing misfolded and aggregated proteins [1] along with selectively clearing damaged organelles, such as mitochondria [2], endoplasmic reticulum [3] and peroxisomes [4]. Autophagy is also involved in the elimination of microorganisms, cell death, tumor suppression [57] and antigen presentation [8].

The first step in the process of autophagy is autophagosome formation. Cytoplasmic constituents and foreign materials are sequestered by a unique membrane called phagophore or the isolation membrane. This membrane has a flat shape and is reminiscent of a Golgi cisterna. Some of the Atg proteins (autophagy-related proteins) gather at the phagophore formation site called "pre-autophagosomal structure" (PAS) [9]. Complete sequestration of the constituents occurs by elongation of the phagophore, results in the formation of autophagosome, which is a double-membrane organelle [10]. In the next step, autophagosomes fuse with lysosomes (in metazoan cells) or vacuoles (in yeast and plant cells). The inner membrane of the autophagosome and the sequestered materials are then degraded by lysosomal/vacuolar hydrolases. These degrading structures are often called "autolysosomes" or "autophagolysosomes." Once the macromolecules or pathogens have been degraded in the autophagolysosome, the resultant amino acids are exported to the cytosol for reuse [11] or the antigens are presented to the T cells to activate the adaptive immune response (Figure 1).

Regulation of autophagy is carried out by autophagy-related genes (ATG genes). Till date 32 ATG genes have been identified in yeast and 16 homologs of these have been characterized in humans [12]. A coordinated action of these genes is essential for the successful formation of an autophagosome. The function of each of these ATG genes is unique and is not complemented. Hence, knockdown or deletion of these genes will result in cell death through either apoptosis or necrosis.

\section{Types of autophagy}

There are three defined types of autophagy: macro-autophagy, micro-autophagy, and chaperone-mediated autophagy CMA. Macro-autophagy is involved in organelle and protein recycling and pathogen clearance. It delivers the cytoplasmic cargo to the lysosomes through autophagosome. Macroautophagy is further classified into "induced macroautophagy" and "basal macroautophagy". The former is involved in amino acid recycling following starvation and during pathogen clearance, while the latter is involved in constitutive turnover of cytosolic components [13].

In micro-autophagy, cytosolic components are directly taken up by the lysosome itself through invagination of the lysosomal 
membrane. The maintenance of organelle size, membrane homeostasis, and cell survival under nitrogen restriction are the main functions of microautophagy. It complements and co-ordinates with macroautophagy, CMA and other self eating pathways to perform these functions [14]. In CMA, targeted proteins are translocated across the lysosomal membrane in complex with chaperone proteins that are recognized by the lysosomal-associated membrane protein 2A (LAMP-2A), resulting in their unfolding and degradation [15].

Autophagy in Innate Immune Mechanisms: Autophagy is rapidly developing into a new paradigm in innate immunology (Figure 2). Its roles in innate immunity have been shown through in-vitro and in-vivo models [16]. Autophagic adapters are capable of identifying Pathogen Associated Molecular Patterns (PAMPs) and Damage-Associated Molecular Patterns (DAMPs). Autophagy inducing PAMPs activate the different Pattern Recognition Receptors (PRRs) such as Toll-like receptors (TLRs), Nod-like receptors (NLRs), RIG-I-like receptors (RLRs) and Sequestosome-like receptors (SLRs) [17]. DAMPs, also called as alarmins, undergo a change in their intracellular localization or are released from damaged cells during cell or tissue injury under sterile or septic conditions [18]. These are identified by autophagic adaptors, thereby activating autophagy. Some examples of alarmins are High mobility group box-1 (HMGB1), Interleukein-1 $\beta$ (IL-1 $\beta$ ), Adenosine Tri-Phosphate (ATP) and DNA complexes [19].
Autophagy possesses anti-inflammatory characteristics. Leaky or depolarized mitochondria release ROS that induce inflammasomes [20]. Basal autophagy prevents this activation of inflammasome by continuously removing the damaged mitochondria [21]. This process is called as mitophagy. If this basal autophagy is impaired, ROS and mitochondrial DNA cause unscheduled inflammasome activation [22]. However, Autophagy can target free pathogens in the cytosol as well as those not killed through the phagocytosis mediated degradation pathway [23]. A study by Nakagawa and colleagues [24] showed that Group A Streptococcus (GAS) that escapes from endosomes into the cytoplasm becomes enveloped by autophagosome-like compartments and is killed upon fusion of these compartments with lysosomes. Apart from GAS, autophagy has also been shown to play a role in the elimination of other bacteria like $M$. tuberculosis, L. monocytogenes and Salmonella species [25].

Recent studies have demonstrated that autophagy in response to an infection by opportunistically invasive commensals like Enterococcus feacalis and invasive intestinal pathogen like Salmonella typhimurium protects the host against invasion. A study from Hooper's lab has shown that autophagy is activated following oral bacterial invasion of intestinal epithelial cells [26]. They have further shown that this autophagic response occurs via the innate immune adaptor protein, MyD88 that is intrinsically present in the epithelial cells. Additionally, they

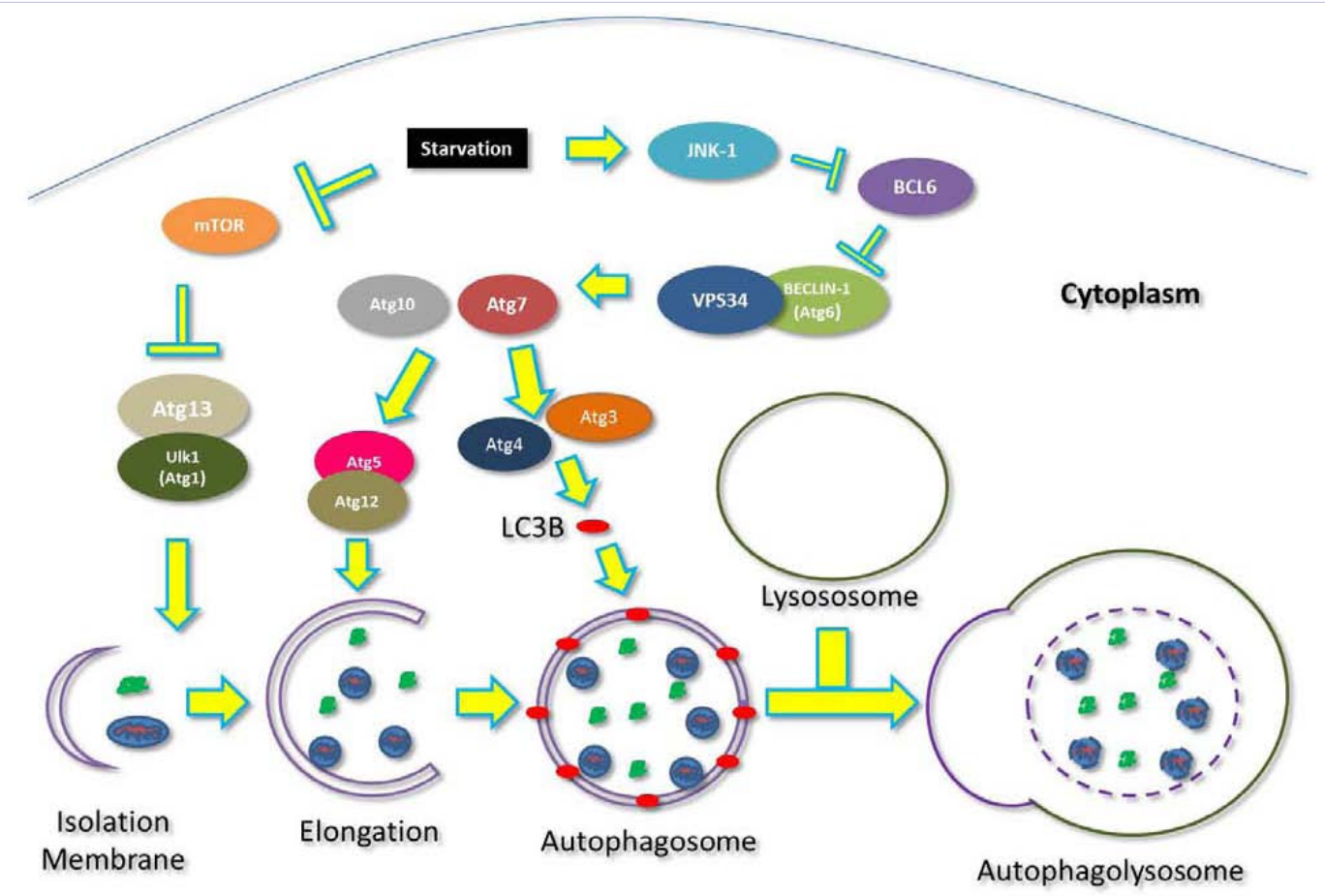

Figure 1: Process of autophagy. An 'isolation membrane' or phagophore is acted upon by a concert of Atg proteins like Atg1 to Atg10, Atg12 to Atg 14 and Atg16. They bring about the extension of the phagophore membrane coupled with sequestration of cytoplasmic components. These results in the formation of a double membrane organelle lined with the autophagy marker protein - Atg8, whose mammalian homologue is known as Microtubule Associated Protein Light Chain 3 (MAP-LC3 or LC3). This autophagosome is then acted upon by Atg15 to bring about the fusion of the outer membrane of the autophagosome with the lysosome. The inner membrane along with the sequestered components are then subjected to degradation by lysosomal enzymes. 


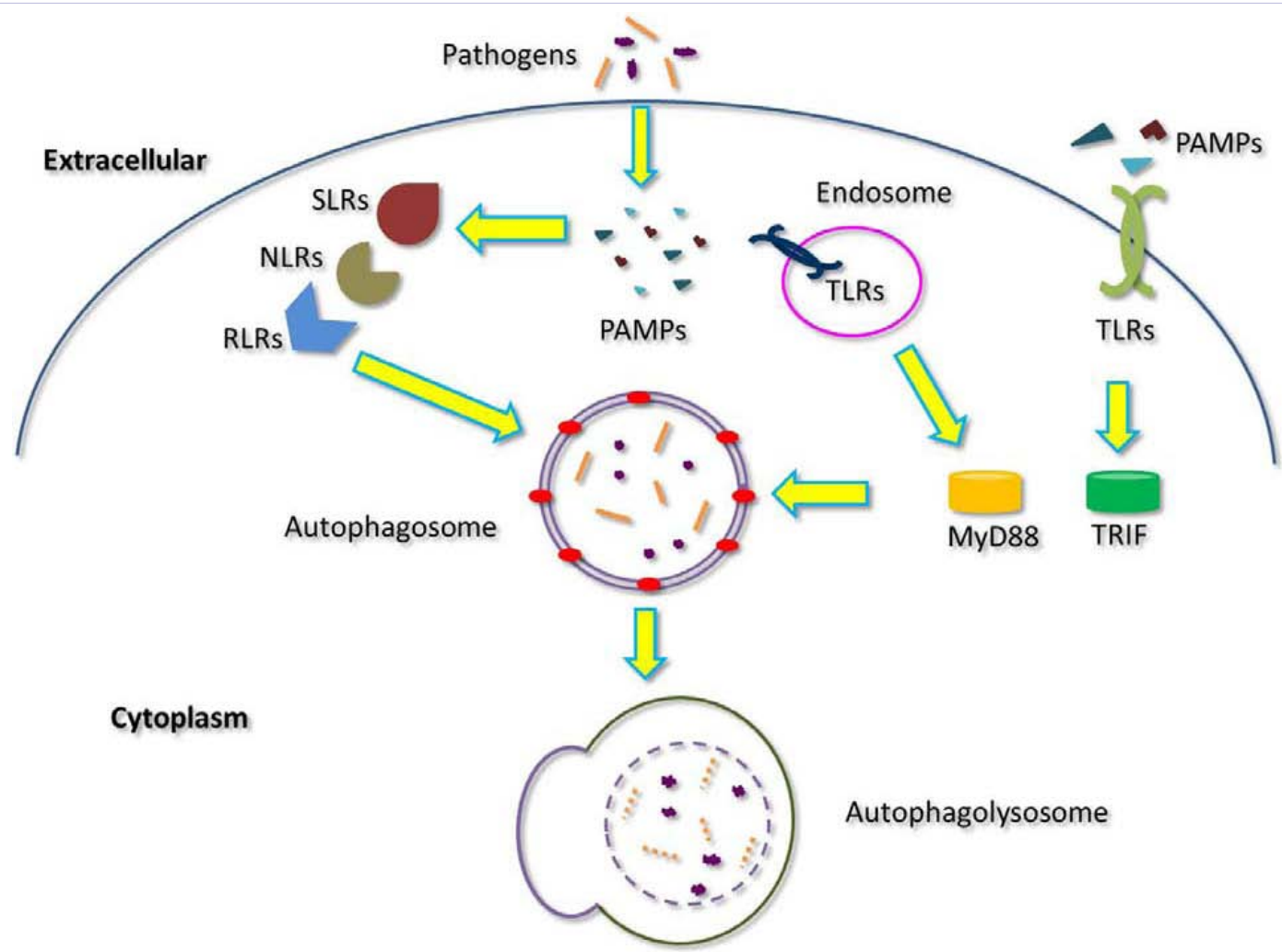

Figure 2: Autophagy in innate immunity. During infection, pathogen and their pathogen associated molecular patterns (PAMPs) are identified by various pattern recognition receptors (PRRs) such as Toll like receptors (TLRs), Nod like receptors (NLRs), RIG-1 like receptors (RLRs) and Sequesterosome like receptors (SLRs). TLRs have been shown to activate autophagy through the MyD88 or TRIF mediated signaling. This activation of autophagy results in sequestering of the intracellular pathogens by autophagosomes, followed by clearance of the pathogen through lysosomal degradation.

showed that mice having the ATG-5 gene conditionally knocked out in intestinal epithelial cells exhibited increased pathogen load and greater dissemination of invasive bacteria to extra-intestinal sites. Thus, autophagy is an important intrinsic mechanism of the epithelial cells to exhibit defense against pathogens in in-vivo conditions [26].

Autophagy in Reproductive Tract Infections: Reproductive tract infections (RTIs) including sexually transmitted infections (STIs) represent a major public health problem especially in the developing countries [27]. The consequences of RTIs/ STIs are numerous and potentially devastating. These include sepsis, ectopic pregnancy, fetal and perinatal death, cervical cancer, infertility, chronic physical pain, emotional distress, and social rejection of women [28]. Some of the most common STIs include Chlamydiasis, Candidiasis, Trichomoniasis, Gonorrhea, Syphilis and Viral Infections like Human Immunodeficiency Virus (HIV), Herpes Simplex Virus (HSV) and Human Papilloma Virus (HPV) infections.

Autophagy and Chlamydiasis: Chlamydia trachomatis (CT) is a human pathogen associated with common STIs. These bacteria enter the host cell and survive within a membranebound vacuole, termed as inclusion body, in which they ensure their successful propagation by avoiding fusion with lysosomes [29]. Non-fusogenity with lysosomes is controlled by the mode of cellular uptake and chlamydial protein factors [30]. Autophagy and its related proteins interact with $C T$ infection in the following ways (Figure 3).

Al-Younes et al. [31] have reported that autophagosome specific stain - Mono Dansyl Cadaverine (MDC) didn't co-localize with chlamydia containing vacuoles. However, autophagy related proteins - MAP-LC3 and calreticulin showed increased accumulation around the pathogen containing vacuoles at an MOI (Multiplicity of Infection) 1. Thus, autophagosome mediated clearance of pathogen may not occur but autophagy related proteins are modulated during infection by $C T$. The role of autophagy during $C T$ infection has been further established using autophagy inhibitors such as 3-Methyl Adenine (3-MA) and synthetic amino acids such as Asparagine, Lysine, and Valine etc. This resulted in smaller $C T$ inclusion bodies and development of defective $C T$. Hence, inhibition of autophagy appears to affect the proliferation of CT. Pachikara et al. [32] observed that autophagy is induced during chlamydia infection after 24 hrs, and hypothesized that unlike the previous observation where autophagy related proteins interacted with the pathogen, autophagy may be induced as a response to excessive nutrient depletion, due to proliferation of $C T$ within the host cells, and not for pathogen clearance. This hypothesis was confirmed by their observation that autophagosome marker protein - Microtubule 


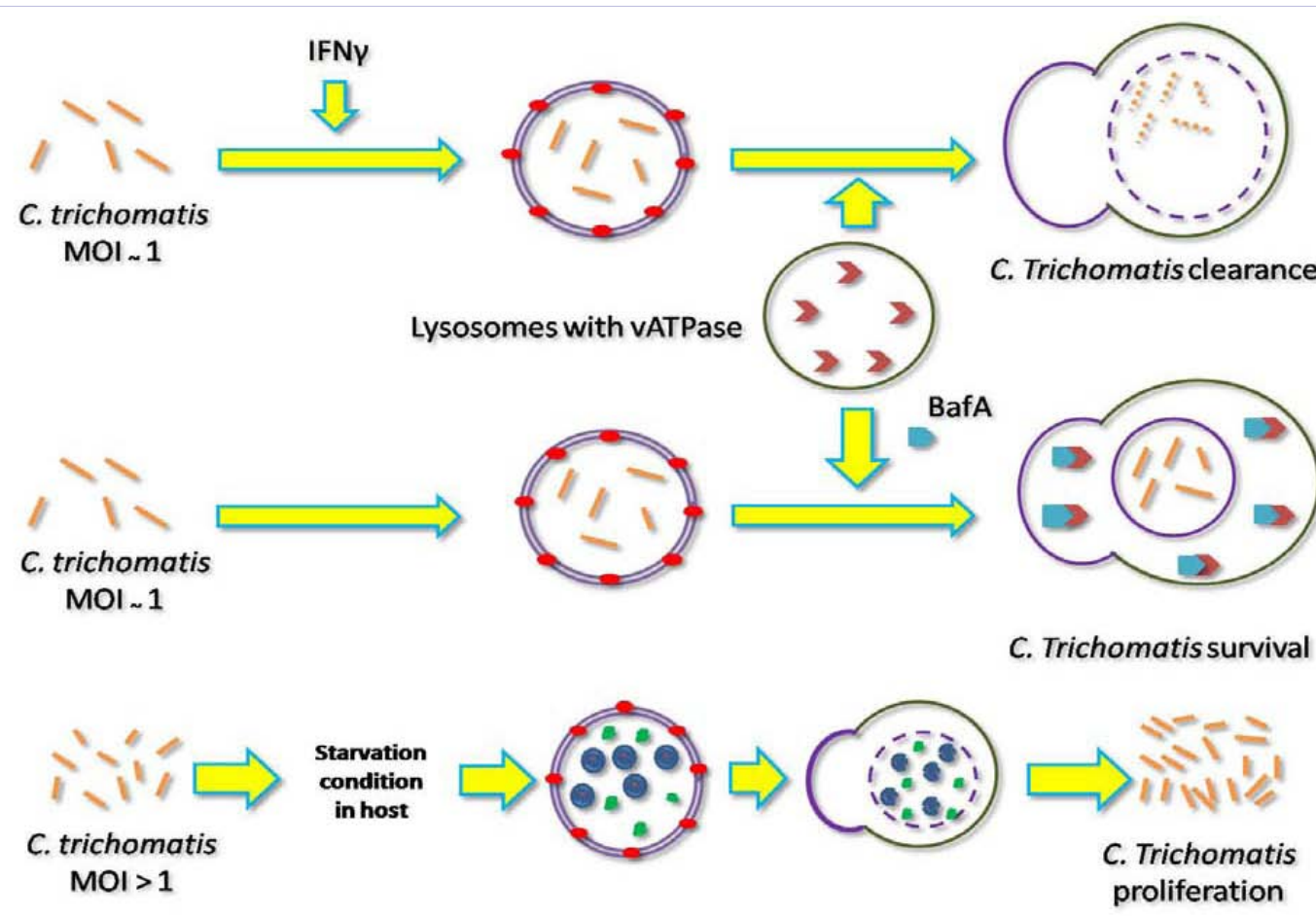

Figure 3: Chlamydia infection and autophagy: Various studies have shown that autophagy plays an important role during Chlamydia trachomatis $C T$ infection. However, the interaction with $C T$ depends on the multiplicity of infection (MOI) of the pathogen. It was observed that during an infection of MOI 1, autophagy mediated degradation of the pathogen was brought about when the cells were treated with IFN $\gamma$. However, at the same MOI, when Bafiliomycin A (BafA) was added to the cells, CT survived as BafA inhibited the lysosomal enzyme vATPase. At higher MOI, it was observed that autophagy was induced but the autophagosomes did not co-localize with the pathogen. Hence, it was thought that autophagy is induced in response to the depletion of nutrients in the host due to the proliferating pathogen.

Associated Protein Light Chain 3 (MAP-LC3 or LC3) did not colocalize with chlamydia containing vacuoles. However, these authors observed that autophagy deficient cells were unable to prevent the growth of pathogen. This suggested that autophagy mediated nutrient recycling was not involved in the growth of pathogens.

vATPase is an enzyme in the lysosome which regulates the function of other lysosomal hydrolases. This would affect the degradation of pathogen through autophagolysosome. When the cells are treated with vATPase inhibitor, Bafiliomycin A (BafA), lysosomal activity is inhibited. Yasir et al. [33] have shown that post infection by $C T$ of wild type Murine Embryonic Fibroblasts (MEFs), treatment with BafA results in the proliferation of $C T$. This proliferation is due to the inhibition of autophagolysome mediated pathogen clearance. Thus, autophagy deficient MEFs should show proliferation of $C T$. However, it was observed that autophagy deficient ATG5 $\%$ MEFs when treated with BafA results in inhibition of $C T$. This opposite effect is because BafA affects cells having proficient and deficient autophagy in different ways.

Chlamydia infection of the host cell is known to induce interferon gamma (IFN $\gamma$ ) mediated immune response. AlZeer et al.[34] have shown that Irga6, Irgd, Irgm2 and Irgm3 proteins accumulate at bacterial inclusion bodies in MEF's upon stimulation with IFN $\gamma$ and this accumulation triggers a rerouting of bacterial inclusions to autophagosomes. These autophagosomes subsequently fuse to lysosomes for the elimination of chlamydia. They have also demonstrated that autophagy-deficient Atg5 1- MEFs were unable to clear the chlamydial pathogens and succumbed to infection even in the presence of IFN $\gamma$. Thus, autophagy may be essential in the clearance of $C$. trachomatis during infection.

LC3B exists in two forms LC3B type 1 that is cytosolic and LC3B type 2 that is incorporated into the autophagosomal membrane. Al-Younes et al., [35] have showed that LC3B that lines the chlamydia vacuole [31] is LC3B type 1 and favors the growth of pathogen. Also, this LC3B type 1 lines the chlamydia membrane even in autophagy deficient cells. However, ATG5 knockout results in increased proliferation of $C$. trachomatis. Hence, this function of LC3B type 1 is independent of autophagy and the autophagy process itself is inhibitory to pathogen proliferation and mediates pathogen killing at lower MOIs.

Autophagy and Candidiasis: Candida albicans (CA) is the most pathogenic species of Candida and is found to be the causative agent in most cases of candidiasis [36]. The most frequent manifestations of genitourinary candidiasis include vulvovaginal candidiasis (VVC) in women, balanitis and balanoposthitis in men, and candiduria in both sexes [37]. Autophagy has been shown to be upregulated in $C A$ infection in multiple studies. Nicola et al., [38] showed that LC3 is localized to vacuoles containing $C A$ during infection of murine macrophages 
by $C A$. They also showed that autophagy is essential in the clearance of pathogen. Disruption of host autophagy in-vitro by RNA interference against ATG5 decreased the phagocytosis of $C A$. Thus, the fungistatic activity of J774.16 macrophage-like cells also decreased. Moreover, mice with a conditionally knocked out ATG5 gene in myeloid cells showed increased susceptibility to intravenous $C T$ infection.

Studies have also implicated the role of autophagy adaptor proteins in the antifungal response of the host cell. Rao et al., [39] have demonstrated that terpenoid phenols like carvacrol exhibit their anti-fungal activity against $C A$ by creating downstream effects similar to the known autophagic inducer, rapamycin that inhibits the activity of major regulator of autophagy, mTOR (mammalian target of rapamycin). The effects include Ca2 ${ }^{+}$bursts, intracellular $\mathrm{pH}$ changes and increased expression of autophagy related genes like ATG1, ATG5, ATG8, etc

Autophagy and Trichomoniasis: Trichomoniasis is the most common non-viral STI caused by the parasitic protozoan Trichomonas vaginalis (TV), with more than 170 million cases annually worldwide. Trichomoniasis leads to serious health outcomes for women, including vaginitis, preterm delivery, infertility, low birth weight, susceptibility to human papilloma virus (HPV) that also leads to cervical cancer and susceptibility to Herpes Simplex Virus (HSV) [40]. In males, TV infection is usually asymptomatic, although urethritis and chronic prostatitis are reported [41]. It has been showed that $T V$ infection is correlated with increased risk of human immunodeficiency virus (HIV) transmission [42] and, lethal prostate cancer [43].

During the time of infection, $T V$ faces competition for nutrients from the commensal flora of the vagina. Huang et al. [44] demonstrated that during conditions of glucose restriction, punctate structures of Atg8, a hallmark indication of autophagy were observed in TV cells. However, these structures were not observed in conditions of glucose abundance. Hence, TV employs autophagy as a survival mechanism during the time of establishing the infection. Once it has access to the host's nutrients, autophagy levels return to the basal state.

\section{Autophagy and viral infections:}

Human Immuno-deficiency Virus (HIV): HIV is the causative agent of Acquired Immuno Deficiency Syndrome (AIDS), and cross-talk between autophagy and HIV occurs at various stages in a host (Figure 4). Kyei et al. [45] have shown that autophagy augments HIV1 yield in macrophages. The Gag protein of HIV1 interacts with LC3B and these are localized on autophagosomes in conditions of basal autophagy. This localization is followed by the proliferation of HIV1 virions within the autophagosome. The involvement of basal levels of autophagy in viral proliferation was further proved by knockdown of basal level autophagy. This knockdown resulted in a decrease in viral load. Therefore, basal level autophagy plays a key role in the proliferation of the virus within the cell. Rapamycin induced autophagy also resulted in increased viral load. Thus, both basal and induced autophagy may support viral replication in macrophages. However, this support is because HIV1 inhibits the degradation capacity of autophagy through Negative Regulatory Factor (Nef) gene. This Nef protein interacts with Beclin1 and inhibits autophagy. Knockout of Nef gene lead to effective autophagy and resulted in decreased viral load.

Autophagy also plays a role in the induction of type two Programmed Cell Death in HIV infected CD4 T cells. Espert et al. [46] have demonstrated that HIV1 envelope proteins X4 and R5 are able to induce autophagy mediated cell death in T-cell cell line MOLT-4. However, this effect was not observed in primary monocytes, which were differentiated into macrophages. Further these authors observed that autophagy mediated cell death in both $\mathrm{T}$ cells and macrophages when they were infected with $\mathrm{X} 4$ and R5 HIV1 tropic strains. Hence, envelope proteins are capable of inducing autophagy mediated cell death even in uninfected T-cells.

Autophagy also plays an important role in lentivirus affected patients, who acquire neurodegenerative diseases. Alirezaei et al. [47] evaluated the number and location of autophagosomal vacuoles in primary monkey and human neurons in response to infection by Simian Immunodeficiency Virus (SIV). They observed that autophagy was inhibited in the neurons on SIV infection. These results were confirmed through the decline in LC3 type 2 and Atg5-Atg12 complex expression levels. However, they found that rapamycin treatment to such SIV infected neurons enhanced autophagic activity and conferred significant protection to the treated neurons. The authors further showed that uninfected neurons when exposed to culture supernatant fluid from SIV-infected microglia resulted in autophagy inhibition in the uninfected neurons.

This effect of SIV infected cells on uninfected cells was further characterized by Van Grol et al. [48]. They have shown that even components of the HIV1 virus inhibits inhibits autophagy in uninfected cells through Src-Akt signaling, which is an autophagy regulator. This inhibition also decreases the overall pathogen clearance ability of the uninfected cells and is not overcome even by using autophagy stimulator- rapamycin. Further, TransActivator of Transcription (Tat) protein of HIV1, receptors like CXCR4, VEGFR1 \& $\beta$-integrins, cytosolic adapters like STAT3 and anti-inflammatory cytokines like IL-10 also play a role in autophagy inhibition in infected cells.

Herpes Simplex Virus (HSV): HSV-1 infection results in varied manifestations such as mild mucocutaneous disease to life-threatening viral encephalitis. Post infection, HSV-1 induces the production of Infected Cell Protein (ICP) 34.5, which plays an important role in the neurovirulence ability of HSV-1 [49]. ICP34.5 inhibits the autophagic response to HSV-1 infection in murine embryonic fibroblasts [50]. This inhibition of autophagy is through dephosphorylation of eukaryotic transcription initiation factor - eIF $2 \alpha$ and physical interaction with autophagy regulatory protein - Beclin-1 [51]. During infection by ICP34.5lacking HSV-1 mutants, the autophagic response depends on the host Protein Kinase R (PKR) signaling pathway and its downstream transcription factor - eIF2 $\alpha$ [52].

Orvedahl et al. [53] have demonstrated that levels of autophagy are affected on infection with wild type or ICP34.5 mutant HSV-1 virus even in Bone Marrow derived Dendritic 


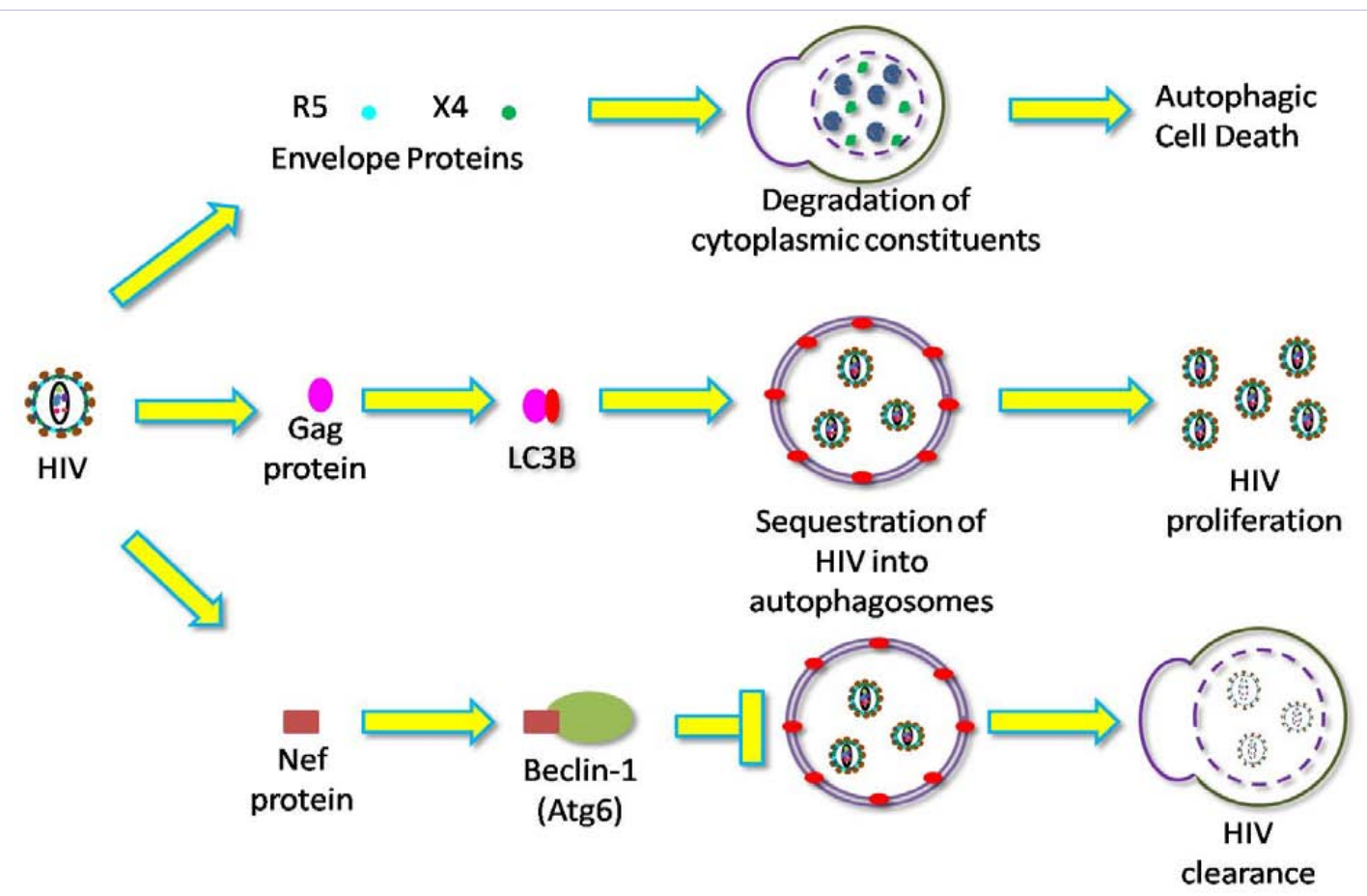

Figure 4: HIV infection and autophagy. Various proteins present in the capsid or on the surface of HIV have been shown to regulate the autophagic response to HIV infection. HIV envelope proteins such as R5 and X4 induce autophagy mediated cell death of the host cells. Proteins Gag and Nef are present in the nucleocapsid of HIV. Gag protein interacts with autophagy marker LC3B and mediates the sequestration of viral particles into autophagosomes. These autophagosomes then do not fuse with lysosomes and provide favorable environment for viral proliferation. Nef protein interacts with Beclin-1 and inhibits autophagy mediated clearance of HIV.

Cells (BM-DCs). They also found that induction of autophagy is not carried out by conventional viral PRRs like TLR2 and TLR9, instead cytoplasmic viral nucleic acid is identified by the cytoplasmic protein-STimulator of INterferon Genes (STING). Viral nucleic acid separates from the viral capsid between 2 to $4 \mathrm{hrs}$ post infection and induction of autophagy is observed around the same time. Further, autophagy is not induced by viral replication and viral entry and hence, cytoplasmic viral nucleic acid is the only viral component that induces autophagy during HSV-1 infection in Bone Marrow derived Dendritic Cells (BMDCs). Inhibition of autophagy results in decreased production of Interferon- $\beta$ (IFN- $\beta$ ) and affects the type I interferon (IFN) response against viral infection.

Yordy and colleagues [54] have shown that viral replication during vaginal infection of ICP34.5 mutant of HSV-1 in wild type and autophagy knockout mice mice is not significantly different. Hence, autophagy in the vaginal epithelial cells of mice may not be involved in limiting the replication of the virus. HSV-1 spreads to the Dorsal Root Ganglionic (DRG) neurons from the vagina. The authors further observed that autophagy played a very important role in the clearance of the pathogen in these neurons. Rasmussen et al., [55] have demonstrated that induction of autophagy differs during HSV1 infection of non-permissive cell types like dendritic cells. They observed that HSV1 infection of BM-DCs led to an upregulation of autophagy even in the presence of ICP34.5. Also, the autophagy response was not through the phosphorylation of eIF2 $\alpha$ but through the expression of IFN genes, which are generally involved in anti-viral response of the cell. They also observed that only viral entry was sufficient to induce this autophagy response and viral gene expression was not required for induction of autophagy.

Human Papilloma Virus: Human papilloma virus (HPV) is a sexually transmitted infection and is the major pathogen in the development of cervical cancer in women [56]. Cervical cancer can be fatal if not identified in the early stages, and prevalence rate of HPV ranges from $7 \%$ to $22 \%$ worldwide [57].

Beclin-1 overexpression results in apoptoic and autophagic death of cervical cancer cell line - HeLa[58]. Zhu et al., [59] have showed that there was a significant down regulation in the expression of Beclin-1 as well as LC3 between normal cervical cells and carcinoma cells. The authors postulated that expression of Beclin-1 and LC3 may have prognostic significance in early stage cervical squamous cell carcinoma. Hence altered Beclin-1 expression levels have been investigated in cervical cancer, cervical intraepithelial neoplasia (CIN) and normal cervical tissues by Cheng et al., [60]. They observed that Beclin-1 expression was significantly different between the three groups, but Beclin-1 expression was negatively correlated with cervical cancer differentiation, lymph node metastasis, recurrence and death.

Potential anti-cancer therapeutics interacts with autophagy in different ways. Metformin [61], Resveratrol [62], Etoposide 
[63], Carboplatin [65] and Paclitaxcel [66]induce autophagy and result in the death of cervical cancer cells through apoptosis. On the other hand, autophagy induction through Cisplatin resulted in prevention of apoptosis mediated death of HeLa cells [64]. The association of HPV infection with the expression of ATPase family AAA domain containing 3A (ATAD3A), which is an autophagy inhibitor was investigated by Chen et al., [67]. The authors found that HPV infection correlated with increased ATAD3A expression and increased drug resistance in cervical cancer patients. They hypothesize that persistent HPV infection may stabilize ATAD3A expression in the cervical carcinoma cells, resulting into inhibition of programmed cell death processes and increased drug resistance.

\section{Conclusion and Future Perspective}

The roles of autophagy have been revealed in the host's innate immune responses and autophagy has been shown to be a critical cellular process that strongly influences inflammation, immunity, and barrier function. Recent in vitro, in vivo, and molecular studies have provided evidence of selective autophagic degradation of bacteria and viruses during infection, also referred to as xenophagy. In this review, the role of autophagy during a number of (RTI's) such as chylamidiasis, candidiasis, trichomoniasis, HIV, HPV and HSV has been discussed. Autophagy response is generally detrimental to the proliferation of pathogens. However, various pathogens such as HIV have developed ways to inhibit the autophagy pathway and thereby prevent their clearance from the cell. Hence, future studies should to be directed towards delineating the molecular details of the autophagy response to therapeutics targeted against these infections. Each pathogen interacts with the autophagy pathway in different ways in different cell types. Therefore, analyzing the molecular details of the autophagy response during other infections such as gonorrhea, syphilis, chancroid and Lympho Granuloma Venereum (LGV) need to be explored.

\section{Acknowledgement}

Authors are thankful to the Director, National Institute for Research in Reproductive Health for encouragement to take up studies in the area of autophagy and host pathogen interaction. The authors acknowledge Department of Biotechnology (DBT) for providing necessary support and NIRRH for providing necessary facilities (NIRRH/Rev/IR/98/2014). AS and DS are grateful to the University Grant Commission (UGC) for providing Junior Research Fellowships.

\section{Contribution to authorship}

KVR and AS formulated the idea for the review. AS, KA and DS conducted the literature review and wrote the first draft of the article. KVR edited the article and all authors contributed to the final version.

\section{References}

1. Nedelsky NB, Todd PK, Taylor JP (2008) Autophagy and the ubiquitinproteasome system: collaborators in neuroprotection. Biochim Biophys Acta 1782 (12): 691-99.
2. Kim I, Rodriguez-Enriquez S, Lemasters JJ (2007) Selective degradation of mitochondria by mitophagy. Arch Biochem Biophys 462(2): 245-253.

3. Mahoney E, Lucas DM, Gupta SV, Wagner AJ, Herman SE, et al. (2012) ER stress and autophagy: new discoveries in the mechanism of action and drug resistance of the cyclin-dependent kinase inhibitor flavopiridol. Blood 120(60): 1262-1273.

4. Kim J, Klionsky D (2000) Autophagy, cytoplasm-to-vacuole targeting pathway, and pexophagy in yeast and mammalian cells. Annu Rev Biochem (69): 303-342.

5. Hippert MM, O’Toole PS, Thorburn A (2006) Autophagy in cancer: good, bad, or both? . Cancer Res 66(19): 9349-9351.

6. Jin S, White E (2007) Role of autophagy in cancer: Management of metabolic stress. Autophagy 3(1): 28-31.

7. Levine B, Deretic V (2007) Unveiling the roles of autophagy in innate and adaptive immunity. Nat Rev Immunol 7(10): 767-777.

8. Klionsky D, Emr S (2000) Autophagy as a regulated pathway of cellular degradation. Science 290(5497): 1717-1721.

9. Suzuki K, Ohsumi Y (2007) Molecular machinery of autophagosome formation in yeast, Saccharomyces cerevisiae. FEBS Lett 581(11): 21562161.

10. Ravikumar B, Sarkar S, Davies J, Futter M, Garcia-Arencibia M, et al. (2010) Regulation of mammalian autophagy in physiology and pathophysiology. Physiol Rev 90(4): 1383-1435.

11. Suriaprianta I, Epple UD, Bernreuther D, Bredschneider M, Sovarasteanu $\mathrm{K}$ et al. (2000) The breakdown of autophagic vesicles inside the vacuole depends on Aut4p. J. Cell Scim 113: 4025-4033.

12. Saitoh T and Akira S (2010) Regulation of innate immune responses by autophagy-related proteins. J. Cell Biol 189(6): 925-935.

13. Mizushima N (2007) Autophagy: process and function Genes. Dev 21(22): 2861-2873.

14. Li WW, Li J, Bao JK (2012) Microautophagy: lesser-known self-eating. Cell Mol Life Sci 69(7): 1125-1136.

15. Glick D, Barth S, Macleod KF (2010) Autophagy: cellular and molecular mechanisms. J Pathol 221(1): 3-12.

16. Oh JE, Lee HK (2013) Autophagy as an Innate Immune Modulator. Immune Network 3(1): 1-9.

17. Levine B, Mizushima N, Virgin HW (2011) Autophagy in immunity and inflammation. Nature 469(7330): 323-335.

18. Sims GP, Rowe DC, Rietdijk ST, Herbst R, Coyle AJ (2010) HMGB1 and RAGE in inflammation and cancer. Annu Rev Immunol 28: 367-388.

19. Deretic V (2011) Autophagy in immunity and cell-autonomous defense against intracellular microbes. Immunol Rev 240(1): 92-104.

20. Zhou R, Yazdi AS, Menu P, Tschopp J (2011) A role for mitochondria in NLRP3 inflammasome activation. Nature 469(7329): 221-225.

21. Nakahira K, Haspel JA, Rathinam VA, Lee SJ, Dolinay T, et al. (2011) Autophagy proteins regulate innate immune responses by inhibiting the release of mitochondrial DNA mediated by the NALP3 inflammasome. Nat Immunol 12(3): 222-230.

22. Deretic V (2012) Autophagy as an innate immunity paradigm: expanding the scope and repertoire of pattern recognition receptors. Current Opinion in Immunology 24(1): 21-31.

23. Virgin HW, Levine B (2009) Autophagy genes in immunity. Nat Immunol 10(5): 461- 470. 
24. Nakagawa I, Amano A, Mizushima N, Yamamoto A, Yamaguchi H, et al (2004) Autophagy defends cells against invading group A Streptococcus. Science 306(5698): 1037-1040.

25. Yuk JM, Yoshimori T, Jo EK (2012) Autophagy and bacterial infectious diseases. Experimental and Molecular Medicine 44(2): 99-108.

26. Benjamin JL, Sumpter R Jr, Levine B, Hooper LV (2013) Intestinal epithia autophagy is essential for host defense against invasive bacteria. Cell Host Microbe 13(6): 723-734.

27. Vishwanath S, Talwar V, Prasad R, Coyaji K, Elias CJ, et al. (2000) Syndromic management of vaginal discharge among women in a reproductive health clinic in India. Sex Transm Infect 76(4): 303-306.

28. Garg S, Sharma N, Bhalla P, Sahay R, Saha R, et al (2002) Reproductive morbidity in an Indian urban slum: Need for health action. Sex Transm Infect 78(1): 68-69.

29. Van Ooij C, Apodaca G, Engel J (1997) Characterization of the Chlamydia trachomatis vacuole and its interaction with the host endocytic pathway in HeLa cells. Infect Immun 65(2): 758-766.

30. Wyrick PB, Brownridge EA (1978) Growth of Chlamydia psittaci in macrophages. Infect Immun19 (3): 1054-60.

31. Al-Younes HM, Brinkmann V, Meyer TF (2004) Interaction of Chlamydia trachomatis serovar L2 with the host autophagic pathway. Infect Immun 72: 4751-4762.

32. Pachikara N, Zhang H, Pan Z, Jin S, Fan H (2009) Productive Chlamydia trachomatis lymphogranuloma venereum 434 infection in cells with augmented or inactivated autophagic activities. FEMS Microbiol Lett 292(2): 240-249.

33. Yasir M, Pachikara ND, Bao X, Pan Z, Fan H (2011) Regulation of chlamydial infection by host autophagy and vacuolar ATPase-bearing organelles. Infect Immun79(10): 4019-4028.

34. Al-Zeer MA, Al-Younes HM, Braun PR, Zerrahn J, Meyer TF (2009) IFN-gamma-inducible Irga6 mediates host resistance against Chlamydia trachomatis via autophagy. PLoS One 4(2): e4588.

35. Al-Younes HM, Al-Zeer MA, Khalil H, Gussmann J, Karlas A, et al. (2011) Autophagy-independent function of MAP-LC3 during intracellular propagation of Chlamydia trachomatis. Autophagy 7(8): 814-828.

36. Pirotta MV, Garland SM (2006) Genital Candida species detected in samples from women in Melbourne, Australia, before and after treatment with antibiotics. J Clin Microbiol 44(9): 3213-3217.

37. Achkar JM, Fries BC (2010) Candida infections of the genitourinary tract. Clin Microbiol Rev 23(2): 253-273.

38. Nicola AM, Albuquerque P, Martinez LR, Dal-Rosso RA, Saylor C, et al (2012) Macrophage autophagy in immunity to Cryptococcus neoformans and Candida albicans. Infect Immun 80(9): 3065-3076.

39. Rao A, Zhang Y, Muend S, Rao R (2010) Mechanism of Antifungal Activity of Terpenoid Phenols Resembles Calcium Stress and Inhibition of the TOR Pathway. Antimicrob Agents Chemother 54(12): 5062-5069.

40. Hobbs MM, Sena AC, Swygard H, Schwebke JR, (2008) Trichomonas vaginalis and trichomoniasis. Sexually Transmitted Diseases, McGrawHill Medical, New York pp: 771-793.

41. Gardner Jr WA, Culberson DE, Bennett BD (1986) Trichomonas vaginalis in the prostate gland. Arch Pathol Lab Med 110(5): 430-432.

42. McClelland RS, Sangare L, Hassan WM, Lavreys L, Mandaliya K, et al. (2007) Infection with Trichomonas vaginalis increases the risk of HIV-1 acquisition. J Infect Dis 195(5): 698-702.
43. Sutcliffe S, Alderete JF, Till C, Goodman PJ, Hsing AW, et al. (2009) Trichomonosis and subsequent risk of prostate cancer in the Prostate Cancer Prevention Trial. Int J Cancer 124(9): 2082 -2087.

44. Huang KY, Chen YY, Fang YK, Cheng WH, Cheng CC, et al. (2014) Adaptive responses to glucose restriction enhance cell survival, antioxidant capability, and autophagy of the protozoan parasite Trichomonas vaginalis. Biochim Biophys Acta 1840(1): 53-64.

45. Kyei GB, Dinkins C, Davis AS, Roberts E, Singh SB et al. (2009) Autophagy pathway intersects with HIV-1 biosynthesis and regulates viral yields in macrophages. J Cell Biol 186(2): 255-268.

46. Espert L, Denizot M, Grimaldi M, Robert Hebmann V, Gay B, Varbanov et al. (2006) Autophagy is involved in T cell death after binding of HIV-1 envelope proteins to CXCR4. J Clin Invest 116(8): 2161-2172.

47. Alirezaei M, Kiosses WB, Flynn CT, Brady NR, Fox HS (2008) Disruption of neuronal autophagy by infected microglia results in neurodegeneration. PLoS One 3: e2906.

48. Van Grol J, Subauste C, Andrade RM, Fujinaga K, Nelson J, et al. (2010) HIV-1 inhibits autophagy in bystander macrophage/monocytic cells through Src-Akt and STAT3. PLoS One 5(7): e11733.

49. Harrow S, Papanastassiou V, Harland J, Mabbs R, Petty R, et al. (2004) HSV1716 injection into the brain adjacent to tumour following surgical resection of high-grade glioma. safety data and long-term survival: Gene Ther 11(22): 1648-1658.

50. Talloczy Z, Jiang W, Virgin HW, Leib DA, Scheuner D, et al. (2002) Regulation of starvation- and virus-induced autophagy by the eIF2alpha kinase signaling pathway. Proc Natl Acad Sci U S A 99(1): 190-195.

51. Alexander DE, Ward SL, Mizushima N, Levine B, Leib DA (2007) Analysis of the Role of Autophagy in Replication of Herpes Simplex Virus in Cell Culture. J Virol 81(22): 12128-12134.

52. Talloczy Z, Virgin HW, Levine B (2006) PKR-dependent autophagic degradation of herpes simplex virus type 1. Autophagy 2(1): 24-29.

53. Orvedahl A, Alexander D, Talloczy Z, Sun Q, Wei Y, et al. (2007) HSV1 ICP34.5 confers neurovirulence by targeting the Beclin 1 autophagy protein. Cell Host Microbe 1(1): 23-35.

54. Yordy B, Iijima N, Huttner A, Leib D, Iwasaki A (2012) A neuron-specific role for autophagy in antiviral defense against herpes simplex virus. Cell Host Microbe 12(3): 334-345.

55. Rasmussen SB, Horan KA, Holm CK, Stranks AJ, Mettenleiter TC, Simon et al. (2011) Activation of autophagy by $\alpha$-herpesviruses in myeloid cells is mediated by cytoplasmic viral DNA through a mechanism dependent on stimulator of IFN genes. J Immunol 187(10): 5268-5276.

56. Pandey S, Chandravati (2012) Autophagy in Cervical Cancer: An Emerging Therapeutic Target. Asian Pacific J Cancer Prev 13(10): 4867- 4871.

57. Kenyon C, Buyze J, Colebunders R (2014) Classification of incidence and prevalence of certain sexually transmitted infections by world regions. Int J Infect Dis 18: 73-80.

58. Wang ZH, Li L, Peng ZL, Duan ZL (2011) Effect of autophagy gene Beclin 1 on the growth of cervical cancer HeLa cells in vitro and vivo. Zhonghua Zhong Liu Za Zhi 33(11): 804-809.

59. Zhu W, Pan X, Li F, Zhang Y, Lu X (2012) Expression of Beclin 1 and LC3 in FIGO stage I-II cervical squamous cell carcinoma and relationship to survival. Tumor Biol 33(5): 1653-1659.

60. Cheng HY, Zhang YN, Wu QL, Sun XM, Sun JR, et al. (2012) Expression of beclin 1, an autophagy-related protein, in human cervical carcinoma and its clinical significance. Eur J Gynaecol Oncol 33: 15-20. 
61. Xiao X, He Q, Lu C, Werle KD, Zhao RX, et al. (2012) Metformin impairs the growth of liver kinase B1-intact cervical cancer cells. Gynecol Oncol 127(1): 249-255.

62. Hsu KF, Wu CL, Huang SC, Wu CM, Hsiao JR, et al. (2009) Cathepsin L mediates resveratrol-induced autophagy and apoptotic cell death in cervical cancer cells. Autophagy 5(4): 451-460.

63. Lee SB, Tong SY, Kim JJ, Um SJ, Park JS (2007) Caspase independent autophagic cytotoxicity in etoposide-treated CaSki cervical carcinoma cells. DNA Cell Biol 26(10): 713-720.

64. Xu Y, Yu H, Qin H, Kang J, Yu C et al. (2012) Inhibition of autophagy enhances cisplatin cytotoxicity through endoplasmic reticulum stress in human cervical cancer cells. Cancer Letters 314(2): 232- 243.
65. Sun Y, Zhang J, Peng ZL (2009) Beclin1 induces autophagy and its potential contributions to sensitize $\mathrm{SiHa}$ cells to carboplatin therapy. Int $J$ Gynecol Cancer 19(14): 772-776.

66. Sun Y, Jin L, Liu J, Lin S, Yang Y, et al. (2010) Effect of autophagy on paclitaxel-induced CaSki cell death. Zhong Nan Da Xue Bao Yi Xue Ban 35(6): 557-565

67. Chen TC, Hung YC, Lin TY, Chang HW, Chiang IP, et al. (2011) Human papillomavirus infection and expression of ATPase family AAA domain containing $3 \mathrm{~A}$, a novel anti-autophagy factor, in uterine cervical cancer. Int J Mol Med 28(5): 689-696. 